\title{
Micro Array Images Segmentation Using a Novel Approach
}

\author{
Pooria Jafari Moghadam Fard ${ }^{1,2}$, M.H. Moradi ${ }^{1,2}$ \\ ${ }^{1}$ Biomedical Engineering faculty of AmirKabir University of Technology, Tehran, IRAN \\ 2 Bioinstrumentation \& Signal processing Lab, AmirKabir University of Technology, Tehran, IRAN
}

\begin{abstract}
DNA micro arrays are more and more frequently used in molecular biology as they can give insight into the relation of an organism's metabolism and its genome. The process of imaging a micro array sample can introduce a great deal of noise and bias into the data with higher variance than the original signal which may swamp the useful information. As imperfections and fabrication artifacts often impair our ability to measure accurately the quantities of interest in micro array images, image processing for analysis of these images is an important and challenging problem. How to eliminate the effect of the noise imposes a challenging problem in micro array analysis. In this paper we implemented a novel algorithm for image sifting which could remove objective noise and simply could remove impulse noise from micro array images. This method could remove objects that smaller than size of grid. We used regular moving grids to sift and remove impulse noise and obtained denoised. In the other hand, this paper describes image processing methods for automatic spotted microarray image analysis. Automatic gridding is important to achieve constant data quality and is, therefore, especially interesting for large-scale experiments as well as for integration of microarray expression data from different sources.

In this paper we have proposed a new method for automatic gridding of micro array images using sum of intensity of columns and rows, and we have developed it to gain a flexible algorithm to determine cells which contain spots of micro array images. By biorthogonal wavelet transform, we increase signal to nose ratio (SNR) of each cells locally. Then with universal threshold we convert the image of each cell to binary image. At last via labeling each of these binary images we have detected pixels of the spots. Lastly, we used Stanford microarray images database as our database and compared results with Genepix and achieved significant improvement.
\end{abstract}

Keywords - Micro array image, enhancement, segmentation, sifting, wavelet

\section{INTRODUCTION}

Micro arrays have become the tool of choice for the global analysis of gene expression. Powerful statistical tools are now available to analyze this expression and to gain an understanding of how changes in gene expression patterns impact biological systems. Currently, several different platforms have evolved from the origin of this imaging technique which goes back to the 1970's [1].
By giving information on the levels of gene expression of thousands of genes at the same time, DNA micro arrays allow researchers, for example, to relate the effect of a disease to particular genes. The basic procedure for a micro array experiment is simply described is as follows. RNA is extracted from a cell or tissue sample and then is converted to cDNA. Fluorescent tags, (usually $\mathrm{Cy} 3$ and $\mathrm{Cy} 5$ ) are enzymatically incorporated into the newly synthesized cDNA or can be chemically attached to the new strands of DNA. A cDNA molecule that contains a sequence complementary to one of the single-stranded probe sequences on the array will hybridize, via base pairing, to the spot at which the complementary reporters are affixed. The spot will then fluoresce when examined using a micro array scanner. The fluorescence intensity of each spot is then evaluated in terms of the number of copies of a particular mRNA, which ideally indicates the level of expression of a particular gene. A schematic diagram for this process created.

Chen et al. [3] describe a segmentation algorithm based on the nonparametric Mann-Whitney test. Given samples of background pixel intensities and putative signal pixels, the test is used to find a set of significant signal pixels.

An important advantage of this approach is its lack of assumptions on spot shape; a drawback is the requirement of a background sample, which is difficult to provide reliably in an automated system. Bozinov et al. [4] have used $\mathrm{k}$ means and other clustering techniques to identify signal pixels in a given image window putatively containing a spot. They use cluster representatives for quantitative analysis rather than spot detection. The approach might be interesting for spot detection purposes but has the same intrinsic initialization problem as the Mann-Whitney segmentation.

Vesanen et al. [2] proposed a spot segmentation and detection method based on a generalized hit-or-miss transform. The approach requires a training sample from which a representative spot shape can be learned.

Yang et al. [5] propose a seeded region growing technique for spot signal segmentation. For grid segmentation of the ApoAl images, they use a manually constructed template. 
In recent years, enhancement and segmentation of microarray images always have been one of the interest fields of image processing papers.

Wei-Bang Chen et al. [6] propose a three-step method for automatic gridding and spot segmentation. They claim that the second step applies a simple, progressive spot segmentation method to deal with inner holes and noise in spots.

Nader Saffarian et al. [7] proposed a new image enhancement approach, which has the ability to improve the gridding results in DNA microarray analysis. The proposed approach is implemented as conditional sub-block bihistogram equalization. The proposed approach first decomposes the signal by a multiresolution transform and then accounts for both the multiscale correlation of the subband decompositions and their heavy-tailed statistics. Hara Stefanou et al. [8] presented a two-stage approach for noise removal that processes the additive and the multiplicative noise component. The proposed approach first decomposes the signal by a multiresolution transform and then accounts for both the multiscale correlation of the subband decompositions and their heavy-tailed statistics. Jinn Ho and WenLiang Hwang [9] integrated the active contour (snake) model and the Fisher criterion to capture, respectively, the boundary and region information of microarray images. They then used the proposed algorithm to automatically segment the spots in the microarray images.

Eleni Zacharia et al. [10] using an original and fully automatic approach for accurately locating a distorted grid structure in a microarray image is presented. The gridding process is expressed as an optimization problem which is solved by using a genetic algorithm. Li Ying et al. [11] constructed the adaptive tensor wavelets for microarray image denoising in terms of an explicit parameterizations of the univariate orthogonal scaling functions. The constructed adaptive wavelet keeps the edge information as possible as. Combining their constructed adaptive wavelet and hidden markov tree model.

In this paper we have proposed a method for automatic gridding of micro array images using sum of intensity of columns and rows, and we have developed it to gain a flexible algorithm to determine cells which contain spots of micro array images. By bi-orthogonal wavelet transform, we increase signal to nose ratio (SNR) of each cells locally. Then with universal threshold we convert the image of each cell to binary image. At last via labeling of each of these binary images we have detected pixels of the spots.

\section{MAterial AND METHOD}

Here, we have implemented a novel algorithm for image sifting which could remove objects with definite size from micro array images [12].
Measurement of spot intensity ratios, which is the ultimate goal of array image analysis, requires the signal regions of the printed spots and also the mapping of the signal regions to logical grid coordinates of printed sequences. Existing software tools commonly use a two-stage approach. First, the print layout positions are established by user interaction or user-initialized semiautomatic methods. This is called "gridding" or "addressing" in the literature. In the second step, signal regions and spot measurements are computed without interaction. In this paper, we try to avoid the interactive preprocessing step as far as possible without losing general applicability to any type of printed array. Noninteractive methods are more suitable in automated systems and help to standardize data analysis.

The essential propose of grid segmentation or gridding is to find approximate positions and sizes of the printed spots these estimations are used to initialize quantitative analysis algorithms for individual spots. In our method we used sum of intensity of columns and rows. Fig.1 shows an original microarray image.

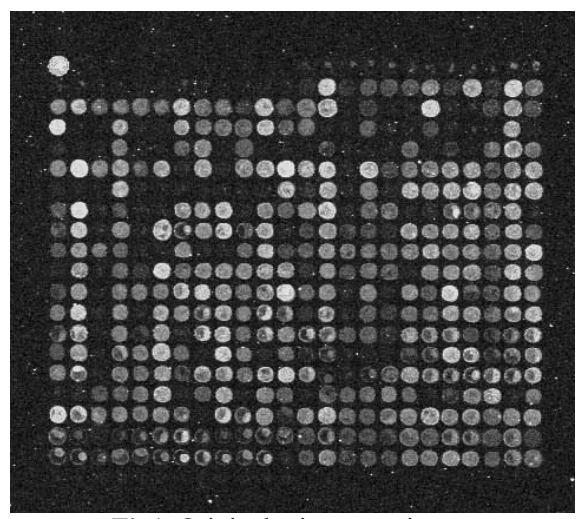

Fig1. Original microarray image

These sums are done on morphologic closed microarray images to obtain two signals as shown in Fig.2.
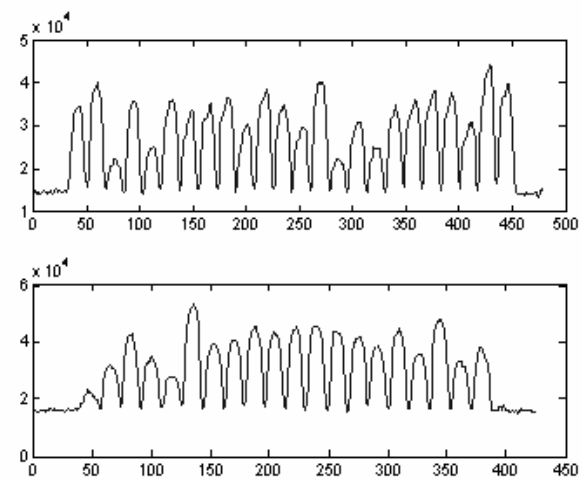

Fig2. Top: Projection of sum of pixel intensity on X-axis Bottom: Projection of sum of pixel intensity on $\mathrm{Y}$-axis

In these signals local minimums show the gap location between spots rows and columns, so grid could align on 
microarray image and separate each individual spot. Fig.3 shows the gridded microarray image by this method.

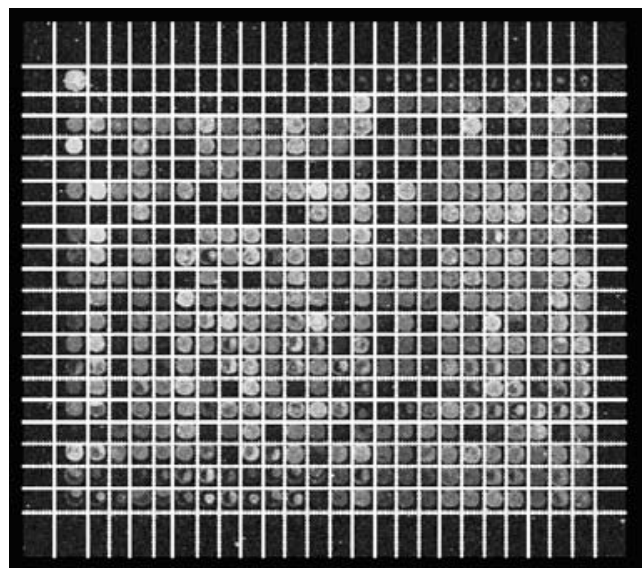

Fig3. Gridded microarray image

Increasing signal to noise ratio (SNR) of each cell is implemented by using approximate coefficients of biorthogonal wavelet transform as shown in Fig. 4. This increase makes it easily to separate pixels which belong to spot from background pixels with simple threshold.

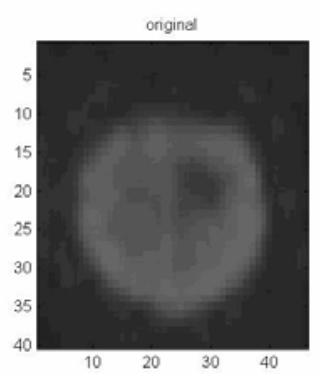

(a)

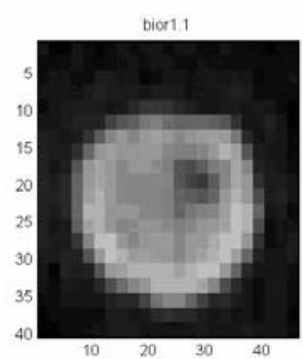

(b)
Fig4. (a) Original spot image (b) Enhanced spot image by bi-orthogonal wavelet

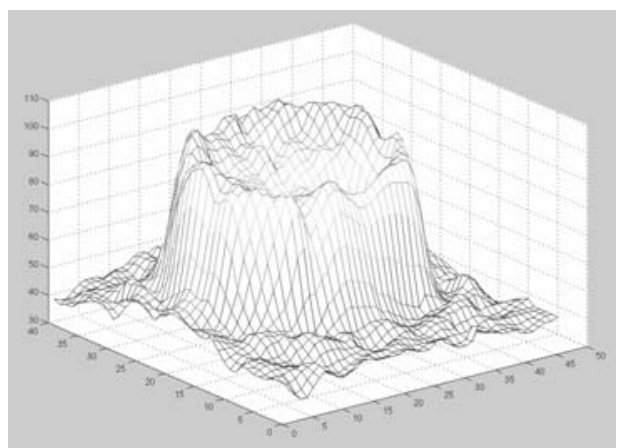

(a)

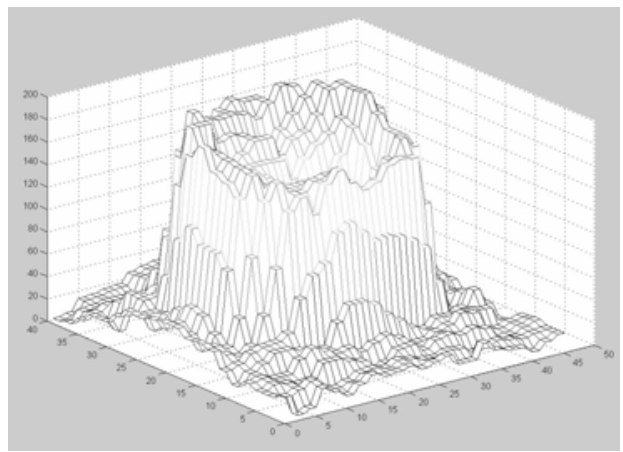

(b)

Fig 5. Applying the bi-orthogonal wavelet, Z-axis shows the brightness level (a) Original spot image (b) Enhanced spot image by bi-orthogonal wavelet

As easily obtain from Fig.5, applying the bi-orthogonal wavelet on each spot considerably increase the SNR. As previously mentioned, we merely used these methods to find the location of each pixel of spot. By applying the biorthogonal wavelet, we obtained over two times improvement on signal to noise ratio.

Also we used threshold to convert the gray scale image of each cell to binary image (black and white). For recognizing the objects in this binary image, we should assign a label to each object. Fig. 6 indicates the scan path for binary image.
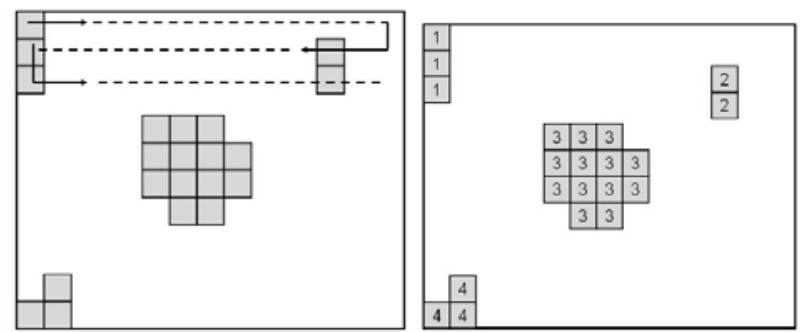

Fig6. Top: scan path for binary image, Bottom: Result of labeling

We find the objects by applying the novel method for scanning the image. Therefore with a method similar to region growing we will find other pixels of the object and let the same label to all of them. For finding other objects we start from the pixel that scanning ended at it to find the next white pixel that has not a label. After the scanning of total image we will have all objects which have their own labels. Based on counting the number of pixel of each object it's determined the biggest one that could be the spot. The criteria that determine this object is a spot or not, are:

1- This selected object should not be grater than $85 \%$ total number of cell pixels.

2- Selected object should not less than $40 \%$ total number of white pixel of related cell.

3- More than two objects with similar size are not accepted. 
After applying these conditions to objects of each cell, we will find the final spot segmented image like Fig.7.

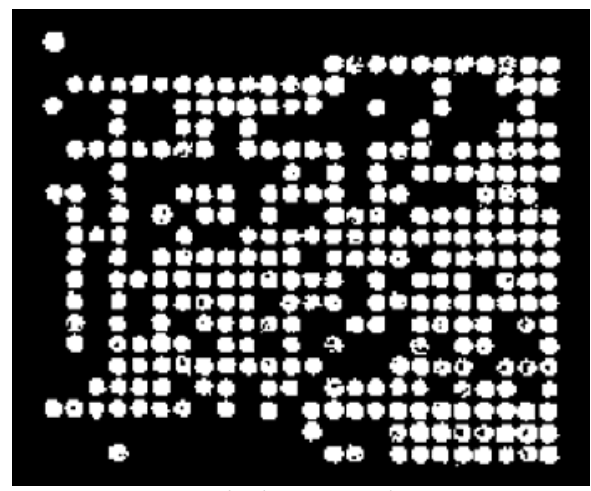

Fig7. Final segmented spots

\section{RESULT}

We used Stanford microarray images database as our database.

The results are compared against Genepix and shown in table 1 as validity table.

\begin{tabular}{|c|c|r|}
\hline Genepix & Current method & \\
\hline 7680 & 7680 & Number of spots \\
\hline 6918 & 6968 & True Positive \\
\hline 82 & 38 & False Negative \\
\hline 20 & 12 & False Positive \\
\hline 662 & 670 & True Negative \\
\hline $98.69 \%$ & $99.45 \%$ & Accuracy \\
\hline $98.82 \%$ & $99.45 \%$ & Sensitivity \\
\hline
\end{tabular}

Table 1. Compare of current method with Genepix

\section{Discussion}

It is well known that micro array technology can monitor thousand of DNA sequences in a high density array on a glass.

As imperfections and fabrication artifacts often impair our ability to measure accurately the quantities of interest in microarray images, image processing for analysis of these images is an important and challenging problem. How to eliminate the effect of the noise imposes a challenging problem in micro array analysis. In this paper we implemented a novel algorithm for image sifting which could remove objects with definite size from macro array images. We have used regular moving grids to sift noise object and obtained clean images for segmentation.

In our method, automatic gridding of micro array images uses sum of intensity of columns and rows, and we have developed a flexible algorithm to determine cells which contain spots of micro array images. We locally increased signal to noise ratio of each cell by bi-orthogonal wavelet transform. Then we convert the image of each cell to binary image with universal threshold. At last via labeling of each of these binary images we have detected pixels of the spots. The advantage of our work is that it makes a trade-off between sensitivity and precision with rapidity. Another high light is that because of using of automatic gridding we are able to apply the algorithm for each cell that makes it possible to select threshold locally which foreground pixels better separate from background pixels.

\section{REFERENCES}

1. E. M. Southern.," Detection of specific sequences among DNA fragments separated by gel electrophoresis", J. Mol. Biol., vol. 98, pp.503-517, 1975.

2. P. Vesanen, "Calibration-free methods in segmentation of cDNA microarray images," in Proc. IS\&T/SPIE 12th Symp. Electronic Imaging Science and Technology, pp. 291-302, 2002

3. Y. Chen, E. R. Dougherty, and M. L. Bittner, "Ratio-based decisions and the quantitative analysis of cDNA microarray images," J. Biomed. Opt., vol. 2, no. 4, pp. 364-374, 1997.

4. D. Bozinov and J. Rahnenführer, "Unsupervised technique for robust target separation and analysis ofDNA microarray spots through adaptive pixel clustering," Bioinformatics, vol. 18, no. 5, pp. 747-756, 2002.

5. Y. H. Yang, M. J. Buckley, S. Dudoit, and T. P. Speed, "Comparison of methods for image analysis on cDNA microarray data," J. Comput. Graph. Statist., vol. 11, pp. 108-136, 2002.

6. Wei-Bang Chen, Chengcui Zhang, and Wen-Lin Liu "An Automated Gridding and Segmentation Method for cDNA Microarray Image Analysis", Proceedings of the 19th IEEE Symposium on Computer-Based Medical Systems (CBMS'06), 2006.

7. Nader Saffarian, Ju Jia Zou, "DNA Microarray Image Enhancement Using Conditional Sub-Block Bi-Histogram Equalization", Proceedings of the IEEE International Conference on Video and Signal Based Surveillance (AVSS'06), 2006.

8. Hara Stefanou, Thanasis Margaritis, Dimitris Kafetzopoulos, Konstantinos Marias, Panagiotis Tsakalides, "Microarray Image Denoising Using a Two-Stage Multiresolution Technique", IEEE International Conference on Bioinformatics and Biomedicine, 2007.

9. Jinn Ho, Wen-Liang Hwang, "SEGMENTING MICROARRAY IMAGE SPOTS USING AN ACTIVE CONTOUR APPROACH", ICIP 2007.

10. Eleni Zacharia, Dimitris Maroulis, "An Original Genetic Approach to the Fully Automatic Gridding of Microarray Images", IEEE TRANSACTIONS ON MEDICAL IMAGING, VOL. 27, NO. 6, JUNE 2008 , page $805-813$

11. Li Ying, Cui Li, "Based Adaptive Wavelet Hidden Markov Tree for Microarray Image Enhancement", The 2nd International Conference on Bioinformatics and Biomedical Engineering, ICBBE 2008.

12. P. Jafari Moghadam, M.H. Moradi, "Image Sifting for Microarray image enhancement", 12th International Conference on Computer Analysis of Images and Patterns, Aug 2007, Vienna, Austria

Author: Pooria Jafari Moghadam Fard

Institute: AmirKabir University of Technology

Country/City: Iran/Tehran

Email: Pooria.jafari@yahoo.com 